\title{
EXPERIENCIAS EN EL TRATAMIENTO DE LA INCONTINENCIA URINARIA DE ESFUERZO
}

\author{
Doctor Rogelio E. Arias *
}

$\mathrm{La}$ incontinencia urinaria de esfuerzo es aquella condición molesta y a veces bochornosa que experimenta una paciente a la cual se le escapa la orina involuntariamente cuando hace algún esfuerzo como el toser, reír, estornudar; o cuando levanta un objeto pesado del suelo o sube un escalón alto.

\section{ETIOLOGIA}

La mayoría de estos casos en nuestro medio tienen su origen en el trauma de la uretra, tejidos parauretrales y cuello de la vejiga ocasionado por el parto. Hay otros casos de origen congénito en los cuales existe defecto en la formación del esfínter vesical o de la uretra, y por último hay un pequeño grupo en el cual el origen se debe a una inervación defectuosa, como son los casos de espina bífida, tabes dorsales, esclerosis múltiple, tumores y trauma de la medula espinal, etc.

En este trabajo se presentan en detalle dos casos; en uno de ellos la incontinencia de esfuerzo se debió a trauma obstétrico, y en el otro a debilidad congénita de los tejidos. Además se añaden en resumen siete casos adicionales operados por el autor desde 1954.

\section{Consideraciones Anatómicas}

Según las observaciones de T. N. A. Jeffcoate y Henry Roberts, la base de una vejiga normal llena es paralela a una línea hipotética que saliendo del borde inferior de la sínfisis pubiana termina en la última vértebra sacral (fig. 1). En ella podemos observar que la uretra forma una "t" con la base de la vejiga, haciendo así un ángulo ante-

* Profesor Asistente de Obstetricia y Ginecología, Facultad de Medicina de la Universidad nacional de Panamá. F.A.C.S., F.A.C.O.G.

Segundo Jefe del Departamento de Obstetricia y Ginecología del Hospital Gorgas, Ancon Zona del Canal de Panamá.

El autor desea dejar constancia de su agradecimiento al profesor T. N. A. Jeffcoate, de la Universidad de Liverpool, por sus atinadas sugerencias y por haber permitido reproducir parte de sus ilustraciones. 
rior y otro posterior (fig. 1: A. A.; A. P.). Durante la micción el piso pelviano se relaja y la base de la vejiga con la parte superior de la vagina $y$

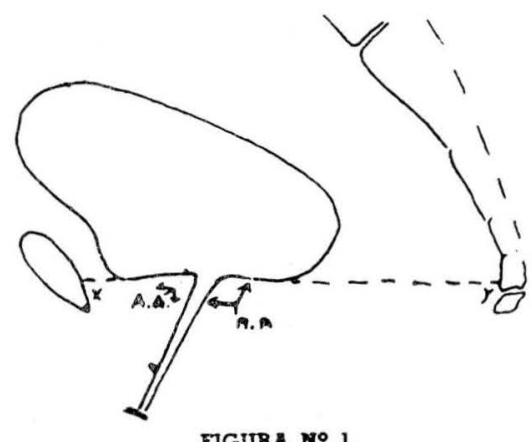

Figura 1. Vejiga normal llena antes de la micción. Nótese el ángulo posterior uretro-vesical (A. P.).

uretra desciende hacia la pelvis, y en esta forma el ángulo posterior vesicouretral desaparece y la parte superior de la uretra se ensancha en forma de embudo (fig. 2: A. P. y E.).

Mientras exista el ángulo posterior vesículo-uretral (A. P.) no habrá incontinencia aun en presencia de prolapso (fig. 3), y es al desaparecer este ángulo cuando la incontinencia de esfuerzo se presenta (fig. 4: A y B). La formación del embudo en la unión uretro-vesical ocurre cuando el ángulo posterior vesico-uretral se borra, y por consiguiente cualquier operación que restituya dicho ángulo curará la incontinencia (fig. 4: C y D).

Algunos de los músculos que intervienen en este fenómeno son el detrusor de la uretra, los elevadores del ano y los transversos del perineo. La fig. 5 muestra la distribución de los músculos voluntarios e involuntarios y el efecto que estos componentes musculares ejercen sobre el ángulo posterior uretrovesical.

En casos leves de incontinencia urinaria de esfuerzo los músculos antes

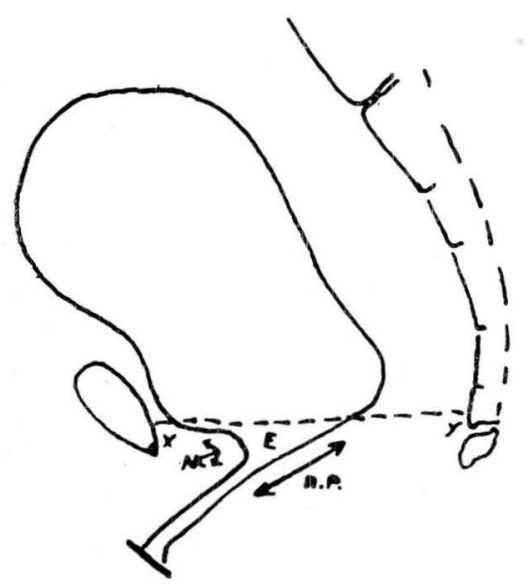

FIGURA No 2.

Figura 2. Vejiga normal durante la micción. Nótese que el ángulo posterior uretro-vesical ha desaparecido $y$ que la base de la vejiga $y$ parte superior de la uretra se han desplazado hacia abajo y hacia atrás.

mencionados pueden mejorar su función mediante ejercicios perineales y vaginales y el uso del perineómetro, alcanzando en muchos pacientes cura completa con una mejoría satisfactoria; sin embargo, el tratamiento por ejercicios fracasa la mayoría de las veces y es entonces cuando se requiere una intervención quirúrgica para corregir este mal tan desagradable.

\section{Tipos de Operaciones}

1. Intervenciones vaginales. 
a) Operación de Kelly, en la cual se hacen repliegues del tejido para-uretral y esfintérico con suturas del estrio. mica con repliegues usando suturas de estrio.

c) Operaciones v a g i n a l e s más complicadas, en las cuales se

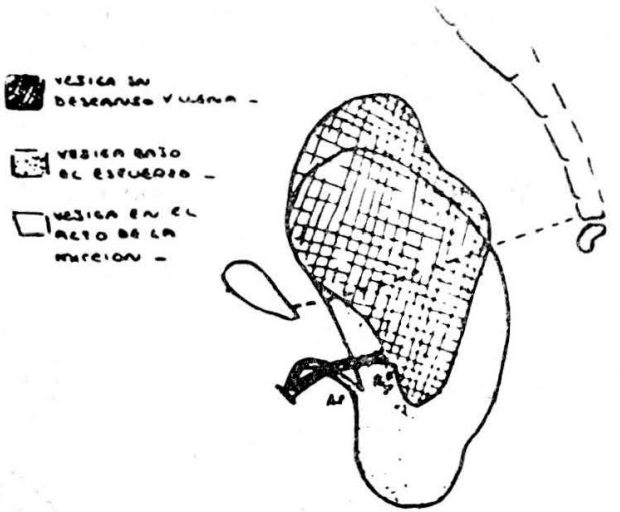

Pavea खง

Vellige de una mulipara os prolapso poro atu incontinoscia dobido a quo ol cisgubo pootericr veaiculowetral (P.S.) es connerpa cisin durasato il osheerso.

Incern nonde do edvess - nótere la areomcia cigl águlo posterict véalco uretral A. $P$.

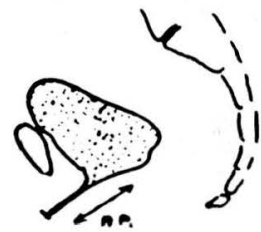

A

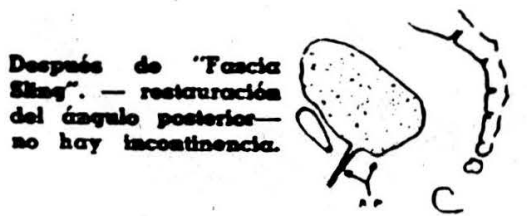

$D$

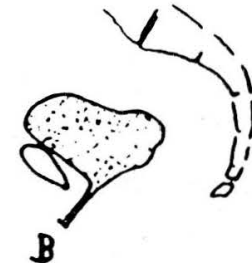

El masurs caso denporo Ia picisther raptad of aragreto postertor of gras crusaro Y la ba comilnezcla os an ac rce.

Despues de "Fasdo 8tiag" - acto do la ab. ciba. epartoncta norind - zecsplo pequecia dra viaction indicada poe la necha dobldo of "gling".

mound in 4.

b) Operación de Kennedy, que consiste en la movilización casi completa de la uretra hasta el cuello de la vejiga para luego hacer una reparación anató- usan diferentes músculos y fascias para la reparación, como la operación de Squier, quien usa los elevadores del ano y perineal transverso. 

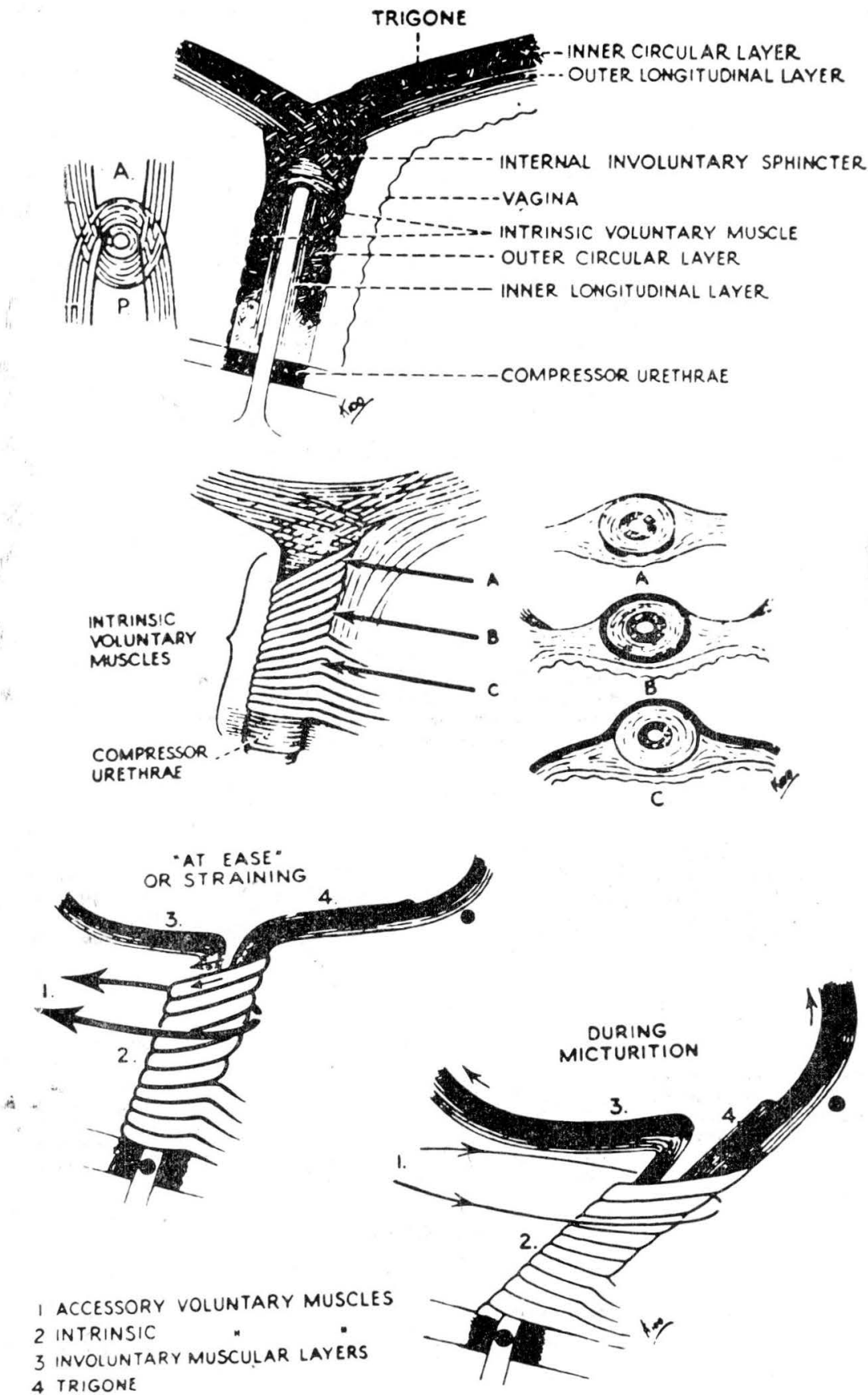

Figura 5. a. Representa la distribución de los músculos involuntarios de la base de la vejiga y uretra. b. Muestra la disposición de los músculos voluntarios intrínsecos de la uretra. c. Representa el efecto que los diferentes componentes musculares ejercen sobre el ángulo posterior uretro-vesical. 
2. Intervenciones suprapúblicas y vaginales conjuntas.

a) Operación de Goebell-Frangenheim-Stoeckel con sus modificaciones como la de Miller y de Aldridge.

3. Intervenciones suprapúbicas.
a) La operación de Marshall-Mar- chetti-Krantz.
b) La operación de Millin.

4. Uso de materiales sintéticos (Mersilene, etc.) que se usan para elevar la base de la vejiga y parte superior de la uretra.

\section{Diagnóstico}

El diagnóstico se hace fácilmente cuando la paciente se presenta con la dolencia de que se le sale la orina involuntariamente al hacer un esfuerzo. $\mathrm{Al}$ reconocimiento ginecológico se observa un descenso de la base de la vejiga y uretra (uretro-cistocele). Cuando se elevan estas estructuras con los dedos y uno hace que la paciente tosa, la orina no se escapa, mientras que si la paciente tose sin estar estas estructuras elevadas, la orina se escapa (prueba de Marshall o de Bonney).

La ausencia del ángulo posterior uretro-vesical se puede demostrar con la uretrocistografía lateral (figs. 6 y 7b). Con esta técnica radiográfica podemos explicarnos el por qué en muchos casos de prolapso no existe incontinencia urinaria de esfuerzo, sencillamente porque el ángulo posterior vesico-uretral está intacto en muchas de estas pacientes (fig. 7a).
La mayoría de los casos de incontinencia urinaria de esfuerzo pueden $\mathrm{cu}$ rarse con una sencilla operación vaginal del tipo de Kelly o Kennedy. Desgraciadamente hay algunos fracasos, $\mathrm{y}$ en estos casos hay que recurrir a intervenciones más complicadas como lo son la operación de Aldridge y la de Marshall-Marchetti-Krantz. Estos fracasos en las intervenciones vaginales se deben casi siempre a la formación de tejidos cicatriciales alrededor de la uretra y esfínter, lo cual impide a este último contraerse y funcionar adecuadamente. Además, hay pacientes que no pueden curarse con repliegues del esfínter vesical y de la uretra (operación de Kelly y Kennedy), como son los casos de ausencia o debilidad congénita del esfínter y de la uretra, y de destrucción de toda o parte de la uretra y la musculatura del esfínter vesical por trauma obstétrico.

Caso número 1, en el cual se practicó la operación de Aldridge. J. H., de 25 años de edad, fue admitida a la Sección de Ginecología del Hospital José Domingo de Obaldía, David, República de Panamá, el 15 de abril de 1947, horas después de haber tenido un parto sumamente traumático debido a distocia céfalo-pélvica. La paciente presentaba:

1. Laceraciones extensas de la vagina, incluyendo los tejidos parauretrales.

2. Laceración de tercer grado del perineo.

3. Fractura de la sínfisis del pubis.

4. Fístula rectovaginal que apareció a los 4 días.

5. Fístula vesicovaginal que apareció a los 8 días. 
Las laceraciones de la vagina se repararon el día de admisión, recibiendo la paciente dos transfusiones y penicilina. Radiografías de la pelvis mostraron fractura de la rama izquierda de la sínfisis del pubis, que se trató con descanso absoluto en cama con el colchón sobre tablas. El 20 de mayo de 1947 se procedió a reparar la fístula rectovaginal y a formar un perineo adecuado con resultado satisfactorio. El 10 de septiembre de 1947 se procedió a reparar operación de Aldridge, siguiendo la técnica que aparece en el libro de Te Linde descrita a continuación: La paciente es puesta en una posición de semidecúbito, de manera tal que se pueda trabajar en la parte inferior del abdomen y al mismo tiempo en la vagina. La parte abdominal de la operación se hace primero para evitar contaminación por la vagina. La incisión se hace del ombligo a la sínfisis del pubis. La fascia del músculo recto se limpia por un centímetro a cada

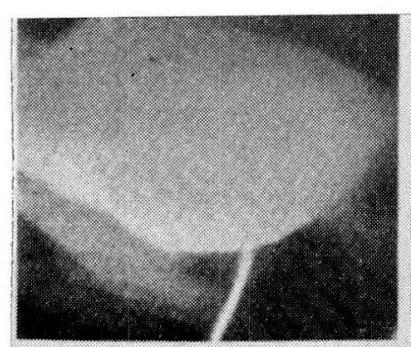

(a)

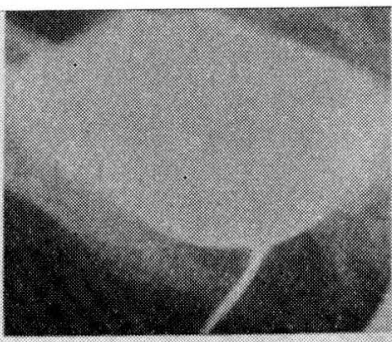

(b)

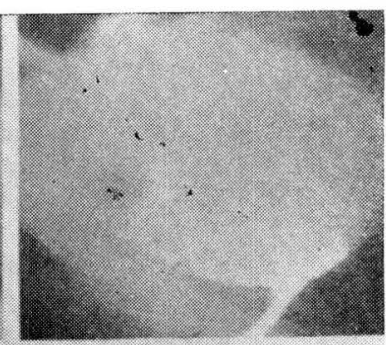

(c)

Figura 6. Uretrocistografía lateral en una multigrávida que sufre de incontinencia urinaria de esfuerzo. a. La paciente en descanso. Los ángulos anterior y posterior uretro-vesicales son normales. b. La paciente haciendo esfuerzo leve pero no lo suficiente para obliterar el ángulo posterior uretro-vesical por completo. c. La paciente haciendo esfuerzo más acentuado. Como se puede apreciar, la base de la vejiga ha descendido más hacia la silla en que está sentada la paciente. El ángulo posterior uretro-vesical ha desaparecido.

la fístula vesicovaginal, la cual se encontraba cerca de la bise de la vejiga, y medía 2 centímetros de diámetro, lográndose el cierre del tracto fistuloso por primera intención. No obstante, la paciente continuaba con una incontinencia urinaria casi total, debido al daño severo ocasionado al esfínter vesical y tejidos parauretrales. La uretra se encontraba dilatada enormemente y admitía fácilmente el dedo índice. El 14 de noviembre de 1947 se le practicó la lado de la línea media. Se libera en el medio un pedazo de fascia, de un centímetro de ancho, dejándose pegado en el extremo interior. El músculo piramidal puede verse en la superficie posterior de la porción inferior de la disección y se deja intacto (fig. 8a). Inmediatamente, para reducir el peligro de infección (fig. 9), se cierra la incisión de la fascia y el tejido subcutáneo de la piel, excepto en el extremo inferior. El cirujano entonces se traslada al campo 
operatorio del perineo. Se hace una incisión corta en el medio de la mucosa vaginal sobre la parte posterior de la uretra, cerca del cuello vesical (fig. 8b) . Se aconseja que se ponga una sonda en la uretra para que se pueda palpar fácilmente durante el curso de la operación. ly y se trae el campo operatorio vaginal a través del túnel retropúbico izquierdo (fig. 8c). Una pinza de Kelly grande se coloca desde arriba en el túnel retropúbico derecho. La punta del pedazo de fascia se agarra y se tracciona con la pinza (fig. 8d). El pedazo de fascia se sutura con seda a la porción inferior

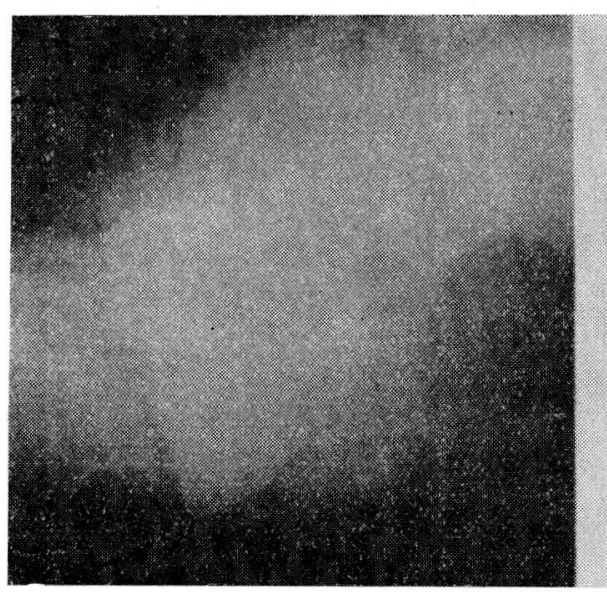

a

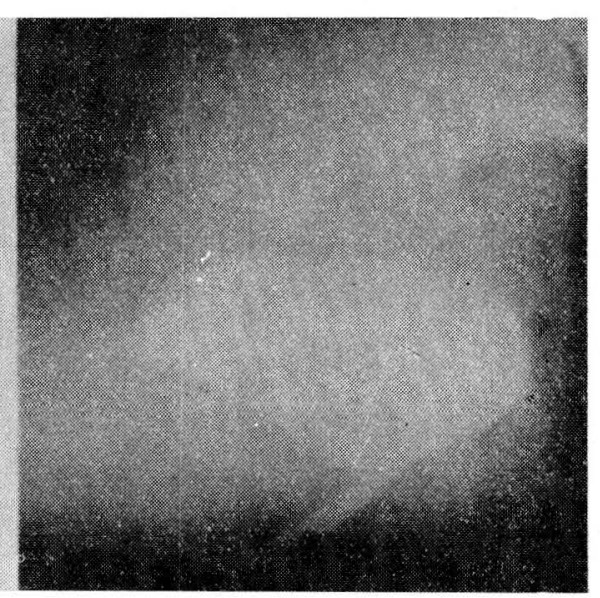

b

Figura 7. a. Prolapso genital sin incontinencia urinaria de esfuerzo. Aunque la uretra superior y base de la vejiga bajan, el ángulo posterior uretro-vesical no desaparece. b. Incontinencia urinaria de esfuerzo en una multigrávida. Al hacer esfuerzo el ángulo posterior uretro-vesical desaparece $y$ la orina se ve escapar hacia la uretra.

Usando una pinza de Kelly se hace un túnel a ambos lados de la uretra, extendiéndolos retropúbicamente hasta que se conecta con la parte inferior de la incisión abdominal. Durante este procedimiento la punta de la pinza debe estar dirigida hacia la sínfisis del pubis para evitar la perforación de la vejiga. De esa manera también se evitan hemorragias severas (fig. 8b). La punta del pedazo de fascia que se había preparado al principio, se agarra con la pinza Kel- de la fascia del recto. Es esencial tener la tensión correcta antes de atarlo. La vejiga se llena con solución salina estéril antes de sacar la sonda, y después que ésta se saca, se hace presión suprapúbica y vaginal, para probar la resistencia del nuevo esfínter. Debe resistir una presión razonable. La parte inferior de la incisión abdominal se cierra.

A esta paciente se le dejó sonda de retención calibre 24, tipo Folley, por 5 días postoperatorios. El asistente y 
el autor trabajaron conjuntamente haciendo uno de ellos la parte abdominal y el otro la parte vaginal, acortando así el tiempo de la operación.
El sexto día de operada se le quitó la sonda, y la paciente pudo tener absoluto control sobre el acto de micción, dándosele salida a los 14 días completa-

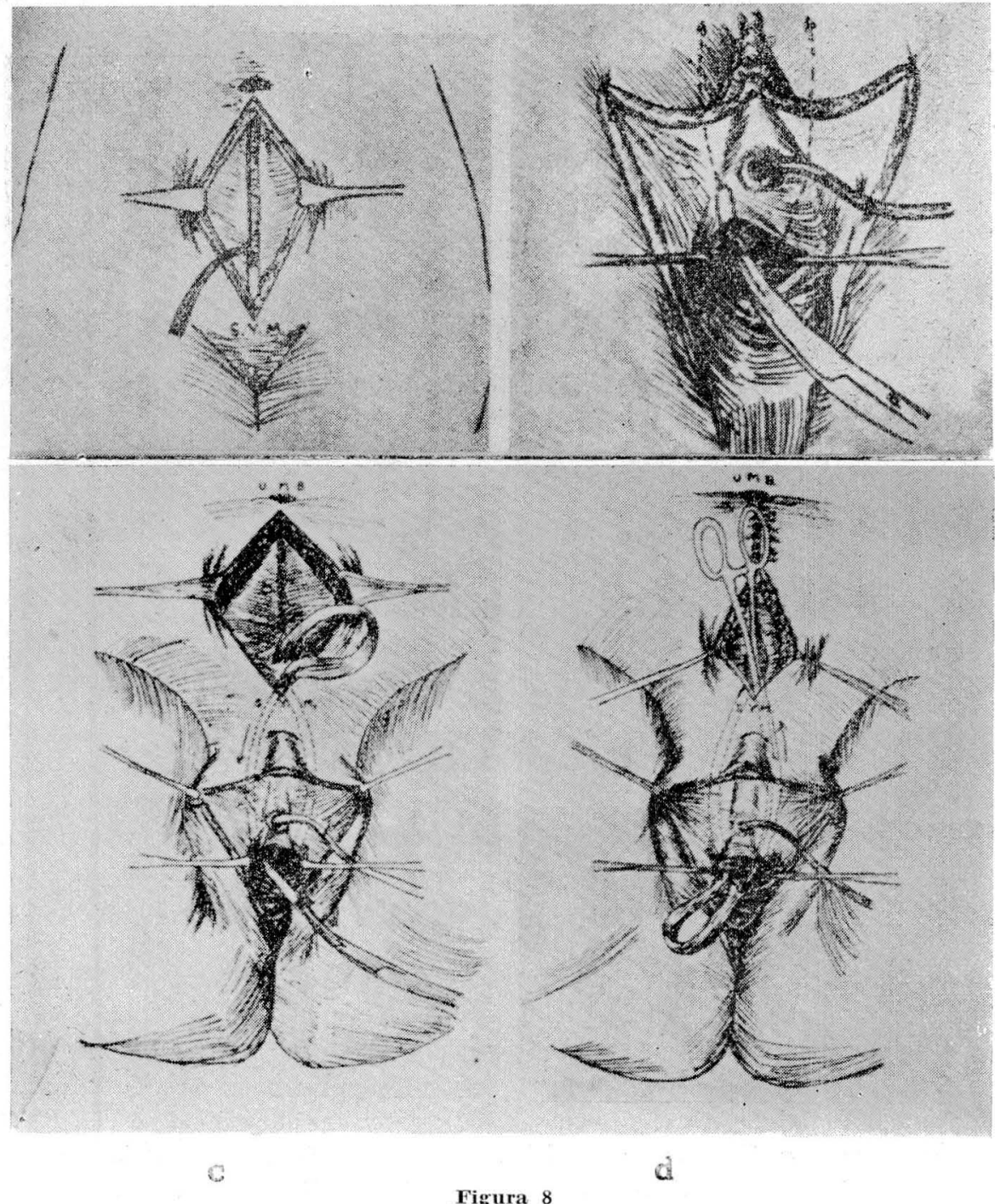

Figura 8 
mente recuperada. En 1949 se le practicó una cesárea para evitar un parto vaginal, y hasta la fecha o sea cerca de 15 años de operada, no ha tenido recidiva de la incontinencia urinaria.

Caso número 2 , en el cual se practicó la operación de Marshall-MarchettiKrantz. I. F., de 45 años de edad, norteamericana, blanca, G II P I (Cesárea) $\mathrm{Ab}$ I. Empezó a tener incontinencia urinaria de esfuerzo mucho antes de 1944, y el 12 de diciembre del mismo año se le practicó una colporrafia anterior, la cual le alivió la incontinencia por 6 meses. El 21 de noviembre de 1949 se le practicó una segunda plastia vaginal para corregir la incontinencia, y esta vez los síntomas se aliviaron solamente por 4 meses. El 16 de julio de 1952 se le practicó una tercera operación vaginal, y de nuevo el alivio de la incontinencia fue de corta duración, 3 a 4 meses. Esta paciente fue readmitida al Departamento de Ginecología del Hospital Gorgas, Ancon, Zona del Canal de Panamá, el 5 de agosto de 1953, adoleciendo de una incontinencia urinaria al esfuerzo, asociada con relajamiento de la pared anterior de la vagina, uretrocele y descenso del cuello vesical, el cual, al ser elevado con los dedos abiertos o con dos pinzas (prueba de Marshall), corregía de inmediato la incontinencia de esfuerzo. En esta ocasión se procedió a suspender la uretra, cuello vesical y vejiga por la vía retropúbica, siguiendo la técnica de Marshall-Marchetti-Krantz que a continuación se describe:

Se insertó una sonda de Folley № 24 en la vejiga. La paciente se colocó en la posición Trendelenburg. Se hizo una incisión supra-púbica extendiéndola has- ta la sínfisis del pubis. La vejiga y la uretra se disecaron hasta 1 centímetro del meatus uretral. El dedo de una ayudante en la vagina ayudó a palpar la sonda. Este último demarcó la uretra y el globo de la región del trígono. Tres suturas de cromico $N^{o} l$ se pusieron equidistantes una de la otra en ambos lados de la uretra. La aguja se enterró en la pared superior de la vagina, cerca de la uretra, y a través de la pared lateral de la uretra, teniendo cuidado de no perforarla (fig. 10). Una sutura similar se practicó a cada lado del esfínter de la vejiga en el ángulo entre el globo y la sonda, después que el globo se aproximó al cuello de la vejiga se elevaron del introito. Se colocaron puntos adyacentes en los lugares indicados de la pared vaginal (fig. 12).

Los extremos de estas suturas se fijaron al periostio del pubis y de los músculos rectos. El ayudante empujando hacia arriba con sus dedos en la vagina antes de atar estos puntos. Se colocaron suturas adicionales en la parte inferior y lateral de la vejiga. Se ataron a la superficie posterior de los músculos rectos (fig. 13). Un drenaje de caucho pequeño se insertó en la herida, y la herida abdominal se cerró de la manera usual. Al inspeccionar la vagina después de este procedimiento se observó que el uretrocele había desaparecido y que la pared vaginal y la uretra habían sido elevados de nivel.

La sonda de Folley se dejó en la vejiga por 7 días. La operación, que duró hora y media, se practicó el 15 de agosto de 1953, y la paciente salió del Hospital 13 días después completamente recuperada y con absoluto control de 


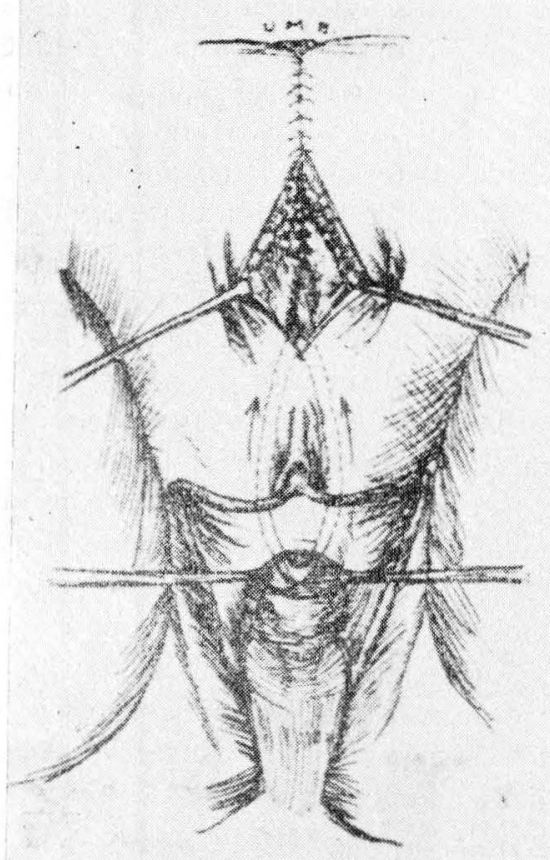

FIGURA No 9.

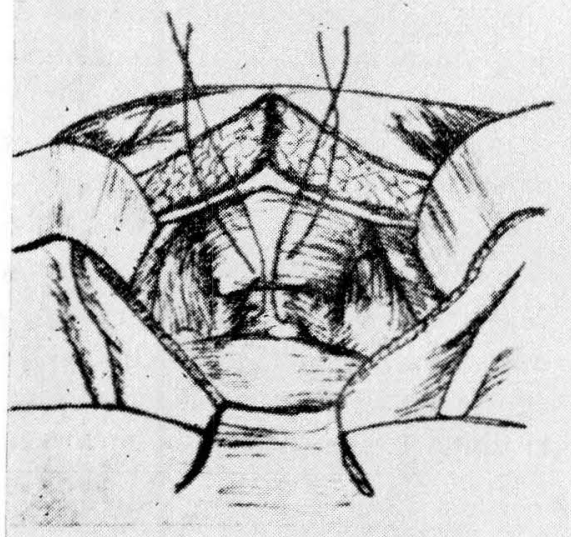

FGURA Ne 10.

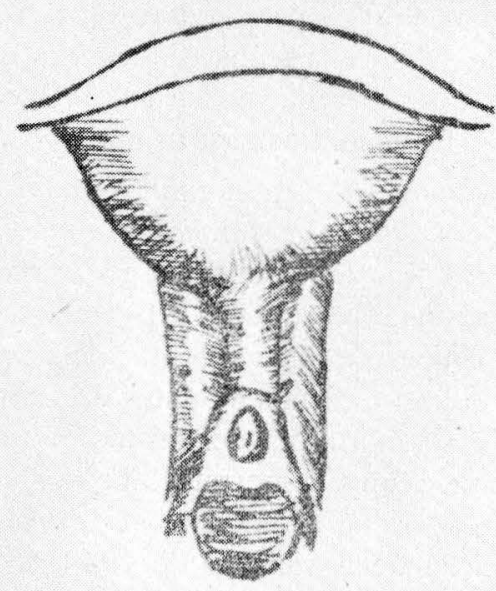

-16U9A No 11.

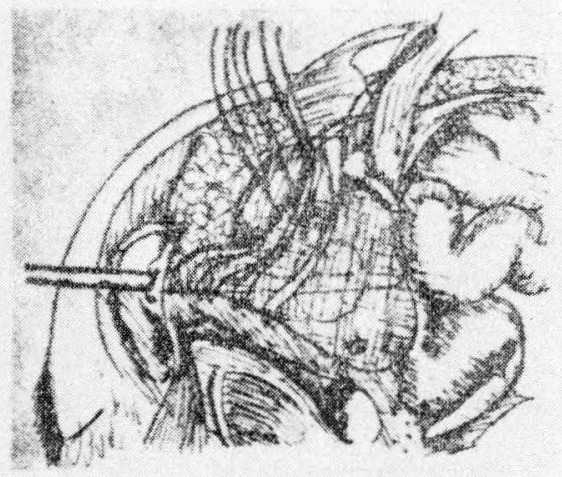

FIGURA No 12.

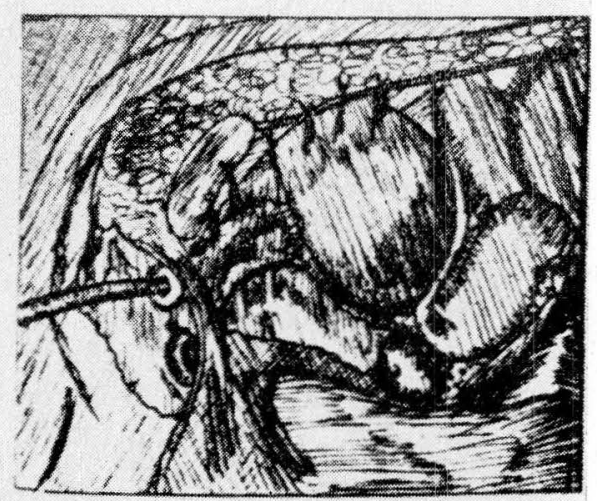

FICURA No 12 
la micción. El curso postoperatorio fue excelente, y la paciente se encuentra en buen estado de salud y sin incontinencia urinaria de esfuerzo.

El resultado que se obtiene después de la operación de Aldridge "sling" rior uretrovesical no desaparece por completo; sin embargo, esta condición no es lo suficiente para causar retención de la orina.

A continuación se muestra una tabla en que aparecen, además de los dos ca-
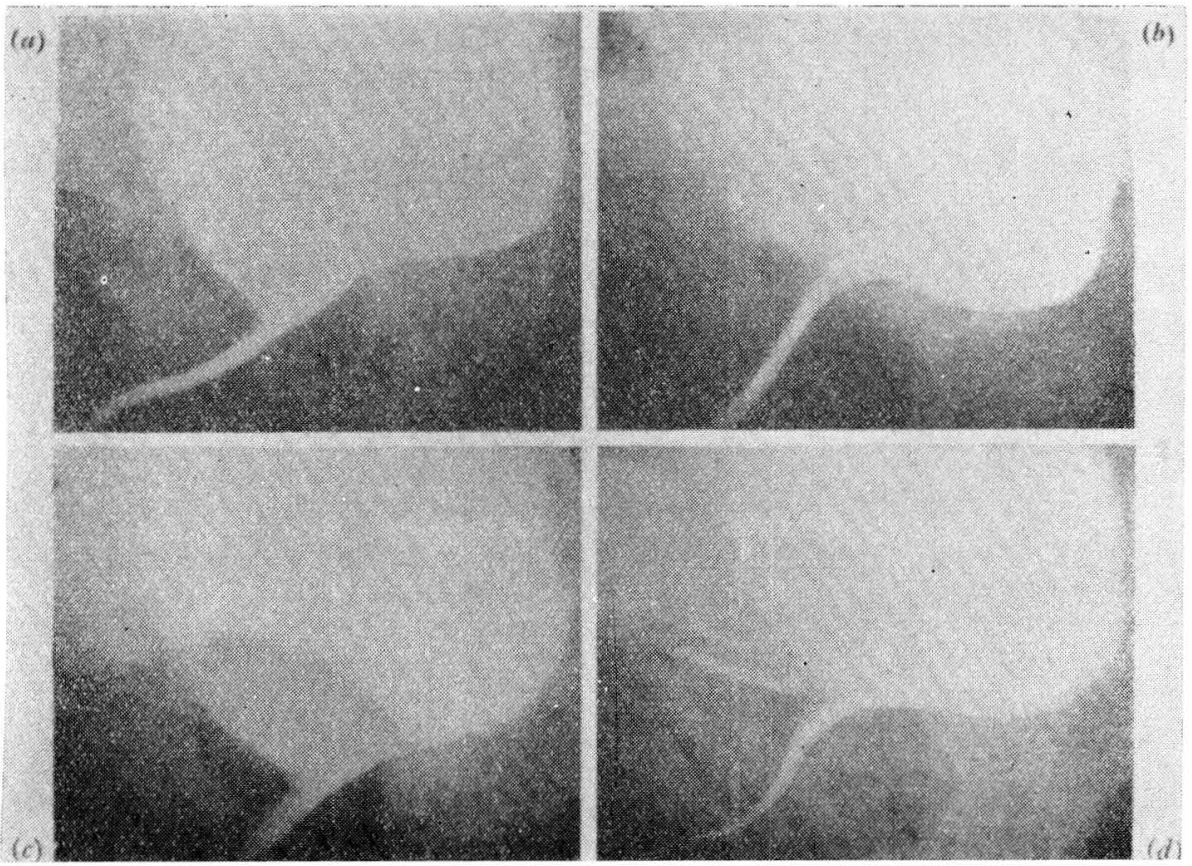

Figura 14. El resultado que se obtiene después de la operación de Aldridge "sling" o hamaca, cuando cura la incontinencia urinaria de esfuerzo. a. Antes de la operación. La paciente haciendo esfuerzo. El ángulo posterior uretro-vesical está ausente. b. Después de la operación. La paciente haciendo esfuerzo. El ángulo ha sido restaurado. c. Antes de la operación. Apariencia normal del acto de la micción. d. Después de la operación, la paciente en el acto de la micción. La vejiga funciona bien pero el ángulo posterior uretro-vesical no ha desaparecido completamente. Esta condición es típica, pero no es lo suficiente para causar retención de la orina.

cuando cura la incontinencia urinaria de esfuerzo se demuestra en la figura $14 \mathrm{~d}$. Después de la operación la vejiga funciona bien, pero el ángulo poste- sos presentados anteriormente, siete casos más que fueron operados por el autor a partir de junio de 1954. 


\begin{tabular}{|c|c|c|c|c|c|c|c|c|c|}
\hline $\begin{array}{l}\text { Caso } \\
\text { No }\end{array}$ & Edad & G. & P. & Fecha de operación & $\begin{array}{l}\text { Indicación } \\
\text { laparotomía }\end{array}$ & Otras operaciones & $\begin{array}{c}\text { Indicación pira } \\
\text { operación }\end{array}$ & $\begin{array}{l}\text { Etiología de } \\
\text { I. U. E. }\end{array}$ & Resultados \\
\hline 1 & 25 & I & O & 14 noviembre 1947 & o & $\begin{array}{l}\text { Fístula R. V. } \\
20-5-47 \\
\text { Fistula V. V. } \\
\quad 10-9-47 \\
\text { Aldridge }\end{array}$ & $\begin{array}{l}\text { Incontinencia } \\
\text { urinaria } \\
\text { completa }\end{array}$ & $\begin{array}{l}\text { Trauma } \\
\text { obstétrico }\end{array}$ & $\begin{array}{l}\text { Bueno } \\
\text { Cesárea } \\
1949\end{array}$ \\
\hline 2 & 45 & II & $\mathrm{I}-\overline{\mathrm{C}}$ & 15 agosto 1953 & $\mathrm{O}$ & $\begin{array}{l}3 \text { Reparaciones } \\
\text { vaginales Ant. } \\
\text { M-M-K }\end{array}$ & $\begin{array}{l}\text { Incontinencia de } \\
\text { esfuerzo }\end{array}$ & $\begin{array}{l}\text { Posible debilidad } \\
\text { congénita de } \\
\text { tejidos }\end{array}$ & Bueno \\
\hline 3 & 32 & $\mathrm{~V}$ & V & 23 junio 1954 & $\begin{array}{l}\text { Dismenorrea } \\
\text { quiste ovárico }\end{array}$ & $\begin{array}{l}\text { D \& C } \\
\text { Resección ovárica } \\
\text { Neurectomía } \\
\text { Presacral } \\
\text { M-M-K }\end{array}$ & $\begin{array}{l}\text { I. U. E. } \\
\text { Cisto-uretrocele }\end{array}$ & $\begin{array}{l}\text { Trauma } \\
\text { obstétrico }\end{array}$ & Bueno \\
\hline 4 & 40 & IV & III & agosto 1954 & $\begin{array}{l}\text { Fibroide } \\
\text { uterino. Meno- } \\
\text { metrorragia }\end{array}$ & $\begin{array}{l}\text { Histerectomia } \\
\text { total } \\
\text { M-M-K }\end{array}$ & I. U. E. & $\begin{array}{l}\text { Trauma } \\
\text { obstétrico }\end{array}$ & Bueno \\
\hline 5 & 46 & II & III & 31 octubre 1956 & $\begin{array}{l}\text { Hiperplasia del } \\
\text { endometrio }\end{array}$ & $\begin{array}{l}\text { Histerectomía } \\
\text { total abdominal } \\
\text { M-M-K }\end{array}$ & $\begin{array}{l}\text { I. U. E. } \\
\text { Cistocele }\end{array}$ & $\begin{array}{l}\text { Trauma } \\
\text { obstétrico }\end{array}$ & Bueno \\
\hline 6 & 32 & VIII & $\overline{V I}$ & 22 julio 1957 & Fibroma uterino & $\begin{array}{l}\text { Histerectomía } \\
\text { total abdominal } \\
\text { Apendectomía } \\
\text { M-M-K }\end{array}$ & $\begin{array}{l}\text { I. U. E. } \\
\text { Cisto-rectocele }\end{array}$ & $\begin{array}{l}\text { Trauma } \\
\text { obstétrico }\end{array}$ & Bueno \\
\hline 7 & 36 & VII & IV & 17 enero 1958 & Retroversión & $\begin{array}{c}\text { Suspensión } \\
\mathrm{M}-\mathrm{M}-\mathrm{K}\end{array}$ & $\begin{array}{l}\text { I. U. E. } \\
\text { Cisto-uretrocele }\end{array}$ & $\begin{array}{l}\text { Trauma } \\
\text { obstétrico }\end{array}$ & Bueno \\
\hline 8 & 60 & III & III & 3 octubre 1958 & $\mathrm{O}$ & $\mathrm{M}-\mathrm{M}-\mathrm{K}$ & $\begin{array}{l}\text { I. U. E. por } \\
28 \text { años }\end{array}$ & $\begin{array}{l}\text { Trauma } \\
\text { obstétrico }\end{array}$ & Malo \\
\hline 9 & 42 & VII & $\mathrm{V}$ & 3 mayo 1961 & $\mathrm{O}$ & $\begin{array}{l}\text { I. Vag. Ant. } \\
\text { M-M-K }\end{array}$ & I. U. E. & $\begin{array}{l}\text { Trauma } \\
\text { abortético }\end{array}$ & Bueno \\
\hline
\end{tabular}




\section{RESUMEN}

1. Se ha definido la incontinencia urinaria de esfuerzo y se han enumerado sus factores etiológicos.

2. En las consideraciones anatómicas de esta enfermedad se considera que el factor más importante es el ángulo posterior uretrovesical, y que mientras este ángulo exista, no habrá incontinencia urinaria de esfuerzo. Los casos de incontinencia urinaria de esfuerzo se curan por cualquier procedimiento quirúrgico que reconstruya dicho ángulo.

3. Se ha hecho una clasificación de las diferentes operaciones, a saber:

a) Operación vaginal. Simple y sencilla, que cura la mayoría de estas pacientes.

b) Intervención vaginal y suprapúbica conjunta.

c) Intervención suprapúbica.

e) Uso de material plástico como la cinta de merselene.

4. Se ha insistido que las operaciones vaginales clásicas pueden curar la mayoría de estas pacientes. En ciertos casos de fracasos en estas operaciones vaginales y en casos de anomalías congénitas o trauma extenso obstétrico se debe recurrir a operaciones más complicadas que tengan por fin la elevación del cuello vesical y la suspensión de la uretra por medio de una hamaca de fascia o por puntos de suspensión al periostio del pubis.
5. Se ha presentado un caso de incontinencia urinaria casi completa debido a un trauma extenso obstétrico operado con éxito por el método de Aldridge.

6. Se ha presentado otro caso de incontinencia urinaria de esfuerzo cuyo factor etiológico se debió probablemente a debilidad congénita de los tejidos, y en el cual después de tres intervenciones vaginales que fracasaron se logró la cura de la incontinencia con la suspensión vesicouretral retropúbica de Marshall-Marchetti-Krantz.

7. Se han presentado también siete casos adicionales en que se practicó la operación de Marshall-Marchetti-Krantz obteniendo buenos resultados en seis de estos siete casos.

\section{COMENTARIOS}

Se considera que los nueve casos presentados en este trabajo, las operaciones efectuadas estuvieron definitivamente indicadas y que tanto la técnica de Aldridge como la de Marshall-MarchettiKrantz dieron excelentes resultados. Por considerarla menos traumática, más sencilla y más rápida se favorece la técnica de Marshall-Marchetti-Krantz, aunque nos proponemos efectuar en el futuro las nuevas técnicas descritas por Te Linde y otros, en las que se usan las cintas de material sintético como el merselene, y esperamos obtener resultados favorables en el uso de este material. 


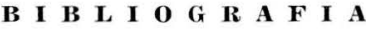

1. Aldridge, A. H.: Amer. Journ. Obst. and Gyn. Vol. 44, 398. Sept. 1942.

2. ARIAS, ROGELIO E.: Experiencias en el tratamiento de la incontinencia urinaria de esfuerzo. Archivos Médicos Nacionales Panameños. Vol. 111. No 4. Oct. Nov. Dic. 1954.

3. CONTE-MENDOZA, H.: La Uretoplastia Retropública de Millin y la Incontinencia Urinaria de Esfuerzo. Archivos Médicos Panameños. Vol. 11. 2: 81-94, 1953.

4. CUESTAS, RAUL: Comunicación personal, 1951 y 1952.

5. CUSAK, WILliams. Comunicación personal, 1952.

6. JEFFCOATE, T. N. A.; ROBERTS, HENRY: Observation on Stress Incontinence of Urine. Am. J. Obst. and Gyn. 64: 721-738, 1952.

7. JEFFCOATE, T. N. A. : Comunicación personal, 1958.

8. JEFFCOATE, T. N. A.: Functional Disturbances of the Female Bladder and Urethra. J. Royal College of Surgeons of Edinburgh. Vol. 7, page 28-47. Oct. 1961.

9. JEFFCOATE, T. N. A.: The Treatment of Stress Incontinence. Postgraduate Medicine, Vol. 21, No 1, Jan. 1957.

10. KEnNEDy, W. T.: Am. J. Obst. and Gyn. 41: 16, 1941.

11. MARSHALL, F. F.; MARCHETTI, A. A., and KRANTZ, K. E.: The Correction of Stress Incontinence by Simple Vesicourethral Suspension. Surg. Gyneco., and Obst. 88; 509, April 1949 .

12. MILlin, T. W.: Retropubic Urinary Surgery. The Williams and Wilkins Company, Baltimore, 1947.

13. ROBERTS, HENRY: Observations on Micturition in the Female. The J. Obst. and Gyn. of the British Empire. Vol. LX, No 5. Oct. 1953.

14. TE LINDE, R. W.: Operative Gynecology. J. B. Lippincott, Philadelphia, pp. 161-162, 1946.

15. STUDDOFORD, W. E.: Transplantation of Abdominal Fascia of the Relief of Urinary Stress Incontinence. Am. J. Obs. and Gyn. 27: 764-775, 1944.

16. WILLIAMS, TIFFANY J., and TE LINDE, R. W.: The Sling Operation of Urinary Incontinence Using Merselene Ribbon. Obst. and Gyn. Vol. 19, No 2. Feb. 1962. 